\title{
Telomerase-Associated Protein TEP1 Is Not Essential for Telomerase Activity or Telomere Length Maintenance In Vivo
}

\author{
YIE LIU, ${ }^{1}$ BRYAN E. SNOW,${ }^{1}$ M. PRAKASH HANDE, ${ }^{2,3}$ GABRIELA BAERLOCHER,${ }^{2}$ VALERIE A. KICKHOEFER, ${ }^{4}$ \\ DAVID YEUNG ${ }^{1,5}$ ANDREW WAKEHAM, ${ }^{1}$ ANNICK ITIE, ${ }^{1}$ DAVID P. SIDEROVSKI, ${ }^{6}$ \\ PETER M. LANSDORP, ${ }^{2,7}$ MURRAY O. ROBINSON,$^{5}$ AND LEA HARRINGTON ${ }^{*} *$ \\ Ontario Cancer Institute/Amgen Institute, Department of Medical Biophysics, University of Toronto, Toronto, Ontario \\ M5G 2C1, ${ }^{1}$ Terry Fox Laboratory, British Columbia Cancer Research Center, Vancouver, British Columbia V5Z 1L3, ${ }^{2}$ \\ and Department of Medicine, University of British Columbia, Vancouver, British Columbia, ${ }^{7}$ Canada; Center for \\ Radiological Research, College of Physicians and Surgeons, Columbia University, New York, New York $10032^{3}$; \\ Department of Biological Chemistry, UCLA School of Medicine and Jonsson Comprehensive Cancer Center, \\ Los Angeles, California 90095'; Department of Pharmacology, UNC-CH School of Medicine, \\ Chapel Hill, North Carolina ; and Amgen Inc., Thousand Oaks, California $91320^{5}$
}

Received 12 June 2000/Accepted 31 July 2000

\begin{abstract}
TEP1 is a mammalian telomerase-associated protein with similarity to the Tetrahymena telomerase protein p80. Like p80, TEP1 is associated with telomerase activity and the telomerase reverse transcriptase, and it specifically interacts with the telomerase RNA. To determine the role of $\mathbf{m T e p} 1$ in telomerase function in vivo, we generated mouse embryonic stem (ES) cells and mice lacking $m$ Tep1. The $m$ Tep1-deficient $\left(m\right.$ Tep $\left.1^{-1-}\right)$ mice were viable and were bred for seven successive generations with no obvious phenotypic abnormalities. All murine tissues from $m$ Tep $1^{-/-}$mice possessed a level of telomerase activity comparable to that in wild-type mice. In addition, analysis of several tissues that normally lack telomerase activity revealed no reactivation of telomerase activity in $m$ Tep $1^{-1-}$ mice. Telomere length, even in later generations of $m$ Tep $1^{-/-}$mice, was equivalent to that in wild-type animals. ES cells deficient in $m$ Tep1 also showed no detectable alteration in telomerase activity or telomere length with increased passage in culture. Thus, mTep1 appears to be completely dispensable for telomerase function in vivo. Recently, TEP1 has been identified within a second ribonucleoprotein (RNP) complex, the vault particle. TEP1 can also specifically bind to a small RNA, vRNA, which is associated with the vault particle and is unrelated in sequence to mammalian telomerase RNA. These results reveal that TEP1 is an RNA binding protein that is not restricted to the telomerase complex and that TEP1 plays a redundant role in the assembly or localization of the telomerase RNP in vivo.
\end{abstract}

Most eukaryotic chromosome ends are maintained by a ribonucleoprotein (RNP) complex called telomerase. Telomerase is a reverse transcriptase that uses an integral RNA component to catalyze the addition of telomeric repeats to the $3^{\prime}$ end of single-stranded telomeric DNA (8).

In many organisms, the telomerase complex is a large (750to $1,000-\mathrm{kDa}$ ) RNP containing an integral RNA, a reverse transcriptase protein subunit, and several associated proteins. The telomerase RNA component provides a template for telomere DNA synthesis, and its essential role in telomerase activity, telomere length maintenance, and chromosome stability has been demonstrated in ciliates, yeast, and mice $(2,14-16$, 29, 39, 42). The telomerase reverse transcriptase (TERT) was first identified in the yeasts Saccharomyces cerevisiae (EST2) and Schizosaccharomycespombe $\left(\right.$ trt $\left.1^{+}\right)$and the ciliateEuplotesaediculatus (p123) $(32,36)$ and subsequently in humans (hTERT) $(12,25,34,36,38)$. Mutations of conserved amino acids within the reverse transcriptase domain of $S$. cerevisiae Est 2 and in human TERT result in the loss of telomerase activity $(6,12,32$, $38,46)$. In rabbit reticulocyte lysates, human telomerase activity is reconstituted by the addition of human TERT (hTERT) and the telomerase RNA $(1,46)$.

In addition to the presumed core telomerase components, consisting of the telomerase RNA and TERT, several proteins

\footnotetext{
* Corresponding author. Mailing address: Ontario Cancer Institute/ Amgen Institute, 620 University Ave., Toronto, Ontario M5G 2C1, Canada. Phone: (416) 204-2231. Fax: (416) 204-2277. E-mail: leah @oci.utoronto.ca.
}

associated with telomerase activity have also been identified. In humans, the "foldosome" proteins hsp90 and p23 and three telomerase RNA binding proteins, dyskerin, L22, and hStau, are each associated with telomerase activity in cell extracts (18, $28,35)$. In $S$. cerevisiae, the Sm protein is necessary for the stability of the yeast telomerase RNA (44), while three other proteins, Est1, Est3, and Cdc13, are dispensable for telomerase activity but are required for telomere length maintenance $(30,33,45)$. In the ciliate Tetrahymena thermophila, two proteins that copurify with telomerase, p80 and p95 (4, 7, 10), bind to telomerase RNA and telomeric DNA, although their precise role in the Tetrahymena telomerase complex is not yet clear (4, 7).

The mammalian homolog of p80, TEP1, is associated with telomerase activity in human, mouse, and rat immortalized-cell extracts $(11,37)$. The amino-terminal 900 amino acids of TEP1, which contain the region homologous to Tetrahymena p80, also interacted with telomerase RNA in an in vivo RNAprotein interaction assay (11). Despite its association with telomerase components, the role of TEP1 in telomerase function is unknown. Recently, TEP1 has also been identified as a component of a large cytoplasmic RNP termed the vault particle (24). The genetic characterization of these proteins is critical to our understanding of the complexity, composition, and regulation of telomerase in vivo. We utilized homologous recombination to disrupt the first mammalian telomerase-associated protein to be identified, mTep1, in mice and embryonic stem (ES) cells and analyzed the effects on telomerase activity and telomere length maintenance. 
A

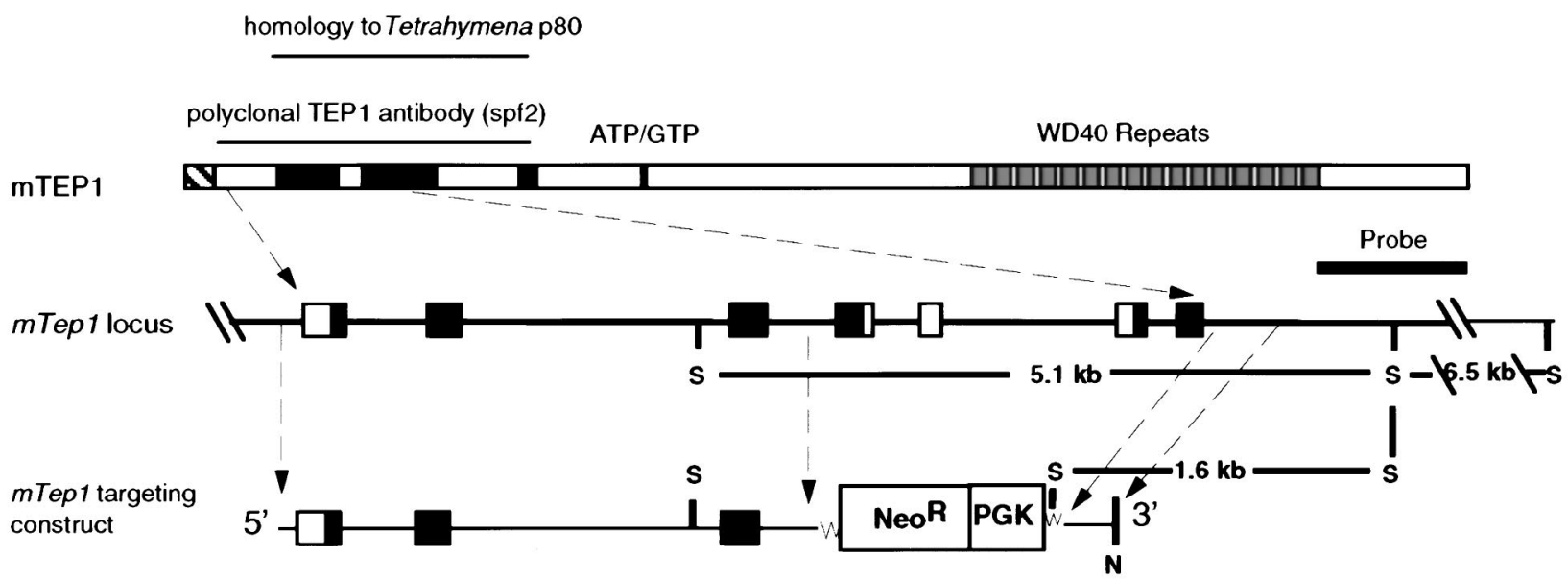

B

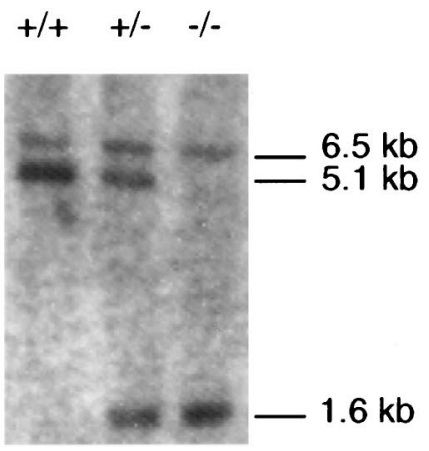

C

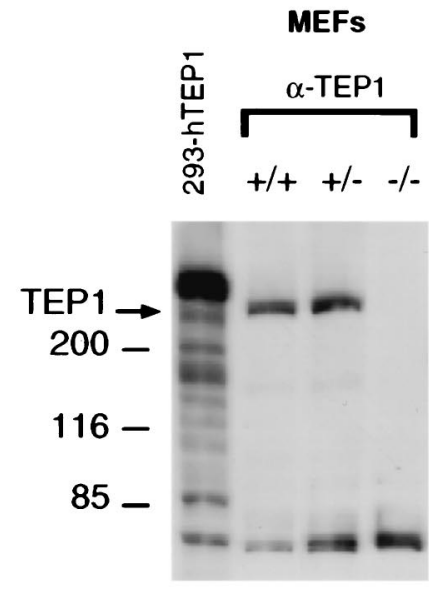

FIG. 1. Disruption of the murine Tep1 gene in ES cells and mice. (A) Schematic representation of homologous recombination of the targeting vector with the endogenous locus. Insertion of a new Spe 1 site into the targeted locus allows the targeted and wild-type alleles to be distinguished by Southern blot analysis with the indicated 3' flanking probe. S, SpeI; N, Not1. (B) Detection of targeted and wild-type mTep1 alleles by Southern blot analysis of DNA from G1 mice. DNA was digested with SpeI and hybridized with the probe shown in panel A. The 5.1-kb SpeI fragment corresponding to the wild-type allele is decreased to $1.6 \mathrm{~kb}$ upon disruption of the locus by integration of the Neo ${ }^{\mathrm{r}}$ gene. (C) Immunoblot of MEF extracts probed with an anti-TEP1 antibody (12). The position of the mTep1 protein is indicated. The first lane contains human 293 cell lysate transfected with a recombinant hTEP1 plasmid (293-hTEP1). The protein species below the 85-kDa protein marker in each immunoprecipitate is not specific to anti-TEP1 antibody.

\section{MATERIALS AND METHODS}

Construction of a murine Tep1 targeting vector. To isolate murine Tep1 genomic fragments, a mouse Tep1 BACMID genomic DNA clone was obtained from Genome Systems Inc. (St. Louis, Mo.) and a full-length mTep1 cDNA probe was used. Two genomic $K p n \mathrm{I} / S p h \mathrm{I}$ fragments (approximately 6.3 and 2.3 $\mathrm{kb}$ ) of the $m$ Tep1 BACMID DNA hybridized to a cDNA probe spanning a region containing the first third of the mTep1 open reading frame. These fragments were subcloned into pSPORT (Stratagene). A targeted construct was designed to replace four exons of $m$ Tep1 with the neomycin resistance gene. In brief, PCR primers (5'-CTCGAGGTTCGTAGGGTCAATGGTGTGTC-3' [sense] and 5'GTCGACATTTCTGTGTTCAAGACAAATCAG-3' [antisense]) were used to amplify a 3.7-kb long-arm fragment from the $6.3-\mathrm{kb}$ mTep1 genomic clone using the Expand long template PCR system (Boehringer Mannheim). Similarly, an 770-bp short arm was amplified from the 2.3-kb mTep1 genomic clone using the PCR primers 5'-TCTAGATAGGTGGCGTTGATCGGTGATCG-3' (sense) and 5'-GCGGCGGCAACCTTTTGAAGAACAACCAATG-3' (antisense).
Targeted disruption of the $\boldsymbol{m}$ Tep 1 gene in ES cells. The $m$ Tep1 targeting vector was linearized with NotI at the short arm and electroporated into E14 ES cells (derived from the R129J strain). After G418 selection $(0.3 \mathrm{mg} / \mathrm{ml})$, homologous recombinants were identified by PCR and confirmed by Southern blot analysis, following published protocols (9). Primer TEP1-1, which is specific for the deleted portion of $m$ Tep1, was used to detect the wild-type allele and primer TEP1-2 was used to detect the mutant allele, while primer TEP1-3 was used to detect both the wild-type and mutant alleles of $m$ Tep1. PCR amplification was carried out with 30 cycles of $94^{\circ} \mathrm{C}$ for $1 \mathrm{~min}, 62^{\circ} \mathrm{C}$ for $1 \mathrm{~min}$, and $72^{\circ} \mathrm{C}$ for $1 \mathrm{~min}$. The sequences of the above primers are as follows: TEP1-1, 5'-CCAGCAGTA TGAGGGTCGTCAGTGG-3'; TEP1-2，5'-GCTAAAGCGCATGCTCCAGA C-3'; and TEP1-3, 5'-CACATGCCTGTCTGGTTCTGTGGAG-3'.

Homologous recombination of the targeting vector with the endogenous locus results in insertion of a novel SpeI site into the mTep1 locus, thus allowing the targeted and wild-type alleles to be distinguished by Southern analysis with a probe corresponding to DNA just $3^{\prime}$ to the short arm. Genomic DNA was 


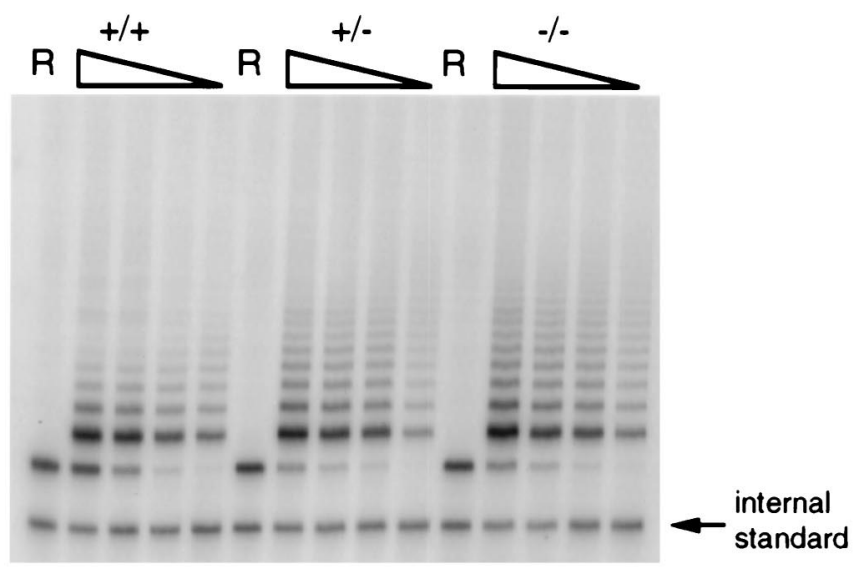

B

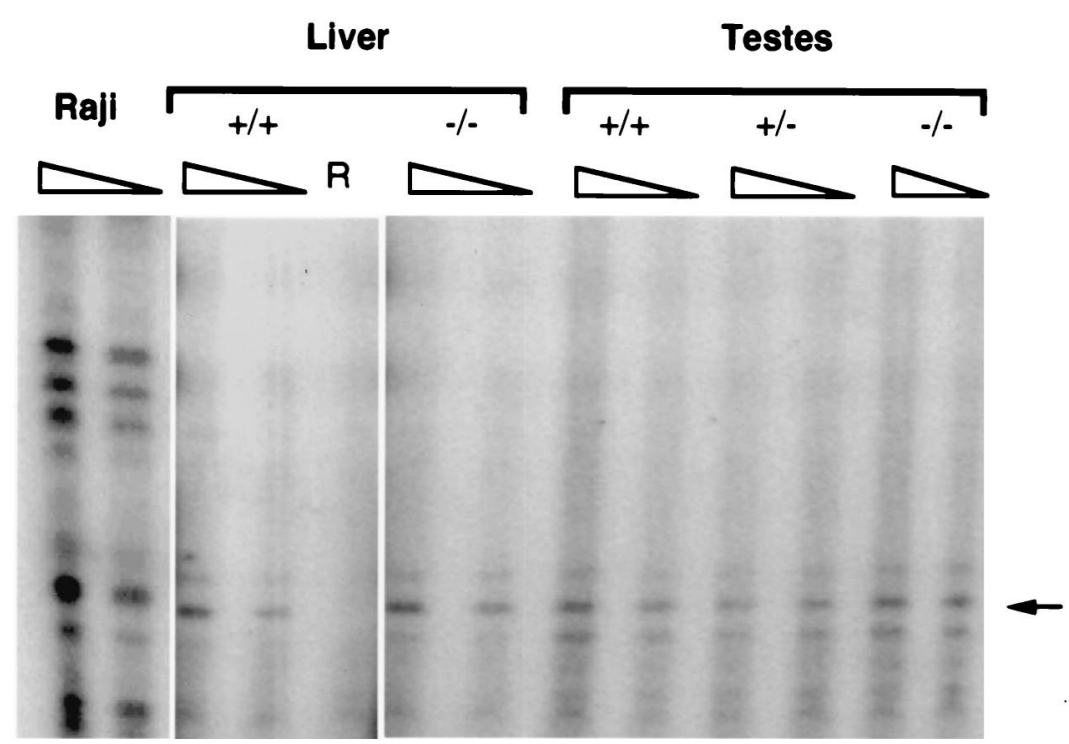

FIG. 2. Telomerase activity of $m$ Tep1-deficient tissues. (A) TRAP was performed for 25 cycles on testis tissue extracts of the indicated genotype. R, RNase A treatment of the sample. An internal PCR standard for TRAP is shown at bottom right. The amounts of testis extract used was as follows: $4 \mu \mathrm{g}$ of extract plus RNase A (wild-type testis), or 4, 2, 1, and $0.5 \mu \mathrm{g}$ of testis extract. (B) The conventional telomerase elongation assay was performed on murine liver and testes. A telomerase-positive human cell extract (Raji) is shown as a positive control. The arrow indicates the position of telomerase elongation products from mouse tissue extract, which were sensitive to RNase A treatment (R). The amount of extract used for each sample was 15 or $7.5 \mu \mathrm{g}$.

digested with SpeI, resolved on an agarose gel, transferred to a membrane, and hybridized to the $m T e p 1$ genomic $3^{\prime}$ flanking fragments.

Generation of $m$ Tep1-deficient mice and ES cells. Chimeric mice were produced by microinjection of three independent $m T e p 1^{+/-}$ES cell clones into E3.5 C57BL/6J blastocysts and transferred to ICR pseudopregnant foster mothers. Chimeric males were mated with C57BL/6J females (Jackson Laboratory). Germ line transmission of the mutant allele was confirmed by PCR and Southern blot analysis of tail DNA from mice with an agouti coat color. The mating strategy for the $m T e p 1^{-1-}$ mice was the same as that used for the generation of the telomerase RNA-deficient mice (2). In brief, the first generation of $m T e p 1^{-1-}$ homozgyous animals were referred to as G1, and the subsequent generations (obtained by mating two $m T e p 1^{-1-}$ animals of an equivalent generation but derived from a different founder) were G2, G3, etc. $m T e p 1^{-1-}$ ES cell clones were generated from G418-resistant $m T e p 1^{+/-}$ES cell clones by culturing with an increased G418 concentration $(4 \mathrm{mg} / \mathrm{ml})$. ES cell culture was carried out as previously described (9).

Cell lysate preparation and telomerase assays. S-100 extracts from cultured cells and freshly dissected mouse tissues were prepared as described previously (41). Cells lysed in a buffer containing 0.5\% CHAPS \{3-[(3-cholamidopropyl)dimethylammoniol-1-propanesulfonate $\}$ were prepared as described elsewhere (26) (Intergen, Inc.). Telomerase activity was assayed using the telomeric repeat amplification protocol (TRAP) assay following the manufacturer's instructions 
(26) (Intergen, Inc.). The standard elongation assay for telomerase activity was performed on S-100 extracts after fractionation on DEAE-agarose columns as described previously $(5,40)$.

Immunoprecipitation analysis of mTep1. Immunoprecipitation analysis using an affinity-purified anti-TEP1 polyclonal rabbit antibody was performed as described previously (12). The specificity of the TEP1 polyclonal antibody was confirmed by Western analysis using cell extracts from human 293 cells overexpressing recombinant hTEP1 and using a glutathione transferase-mTEP1 recombinant purified fusion protein (12).

Telomere length measurements by FISH. The average telomere fluorescence at chromosome ends in splenocytes, thymocytes, and ES cells was measured by using fluorescence in situ hybridization (FISH) combined with flow cytometry (Flow-FISH) (43), with minor modifications. A telomere-specific fluorescein isothiocyanate-conjugated (CCCTAA $)_{3}$ peptide nucleic acid probe $(0.3 \mu \mathrm{g} / \mathrm{ml})$ (Perseptive Biosystems) was employed. Telomere fluorescence is expressed as molecules of equivalent soluble fluorochrome (MESF) (13).

Metaphase spreads, FISH and image analyses of ES cells, and mouse embryo fibroblast (MEF) cultures were performed as described elsewhere $(2,47)$. The Cy-3-labeled (CCCTAA) 3 peptide nucleic acid was used as a probe.

\section{RESULTS}

Targeted disruption of $\boldsymbol{m T e p} 1$ genes. The mTep1 targeting strategy was designed to disrupt a region of mTep 1 that is required for binding to the murine telomerase RNA in a yeast three-hybrid interaction assay (11) (Fig. 1A). Three separate heterozygote $m$ Tep1 $\left(m\right.$ Tep $\left.1^{+/-}\right)$ES cell clones were used to generate $m$ Tep $1^{+/-}$founder mice (data not shown). One of the $m T e p 1^{+/-}$ES cell clones was used to generate three nullizygous $m$ Tep 1 ( $m$ Tep $1^{-/-}$) clones. Different $m$ Tep $1^{+/-}$founders were bred together to generate $m T e p 1^{-1-}$ mice (Fig. 1B). The mTep $1^{-1-}$ mice were designated as generation $1(\mathrm{G} 1)$, and the progeny of G1 cousins were defined as G2, et cetera, as described previously (2). Seven generations (to G7) of $m$ Tep1 $1^{-/-}$ mice were fertile and showed no obvious phenotypic defects compared to wild-type mice. Northern analysis of poly $(\mathrm{A})^{+}$ mRNA from several $m$ Tep $1^{-1-}$ mouse tissues showed no detectable transcripts upon hybridization to a full-length mTep1 cDNA probe (data not shown). Analysis of cell extracts prepared from $m$ Tep $1^{-1-}$ G1 MEFs with an affinity-purified anti-TEP1 rabbit polyclonal antiserum (spf2) revealed no detectable mTep1 protein in lysates or anti-TEP1 immunoprecipitation from mTep1-deficient mouse tissues (Fig. 1C and data not shown).

mTep1 is not essential for telomerase catalysis in vivo. To determine the role of mTep1 in telomerase catalysis, we examined $m$ Tep 1-deficient ES cells, MEF cultures derived from G1 $m$ Tep $1^{-1-}$ embryos, and several tissues from $m$ Tep $1^{-1-}$ mice (up to G3) for telomerase activity. In both the conventional telomerase elongation assay and the PCR-based telomerase assay (TRAP) (26), all three independently derived mTep $1^{-1-}$ ES cell clones possessed levels of telomerase activity similar to those in the $m T e p 1^{+/-}$clone and the parental line (data not shown). We also tested whether telomerase activity was altered in tissues from $m$ Tep $1^{-/-}$mice. Telomerase activity was not significantly altered in testes, liver, kidney, lung, or thymus tissue from $m$ Tep $1^{-/-}$mice compared to wild-type mouse tissues (Fig. 2A and Table 1). Subsequent analysis of several tissues that normally lack telomerase activity, including brain, skin, heart, and spleen $(2,41)$, revealed no reactivation of telomerase activity in these tissues from $m$ Tep $1^{-1-}$ mice (Table 1 and data not shown). In MEFs derived from G1 $m$ Tep $1^{-1-}$ embryos, there was also no significant change in telomerase activity compared to that in the $m$ Tep $1^{+/-}$and wild-type MEFs (Table 1 and data not shown). In the conventional telomerase assay, we did not detect any changes in telomerase activity levels in liver or testis extracts from mTep $1^{-/-}$mice (Fig. 2B).

The role of $\mathrm{mTep} 1$ in telomere length regulation in vivo. Mice and ES cells disrupted in the telomerase RNA compo-
TABLE 1 . Telomerase activity in tissues analyzed by TRAP assay

\begin{tabular}{llll}
\hline \multicolumn{1}{c}{ Extract } & \multicolumn{3}{c}{ Level of activity } \\
\cline { 2 - 4 } \multicolumn{1}{c}{ source } & \multicolumn{1}{c}{ Wild type } & \multicolumn{1}{c}{+-} & $-/-$ \\
\hline ES cells & Strong & Unchanged & Unchanged \\
Primary MEFs & Strong & Unchanged & Unchanged \\
Testes & Strong & Unchanged & Unchanged \\
Liver & Strong & Unchanged & Unchanged \\
Thymus & Strong & Unchanged & Unchanged \\
& & & \\
Lung & Weak & Unchanged & Unchanged \\
Kidney & Weak & Unchanged & Unchanged \\
& & & \\
Heart & Undetectable & Undetectable & Undetectable \\
Brain & Undetectable & Undetectable & Undetectable \\
Skin & Undetectable & Undetectable & Undetectable \\
Spleen & Undetectable & Undetectable & Undetectable \\
\hline
\end{tabular}

nent lack telomerase activity and undergo telomere attrition in vivo (2, 16, 29, 39). In S. cerevisiae, EST1, EST3, and CDC13 are not required for telomerase activity (31); however, mutation or disruption of these genes results in progressive telomere loss $(30,31)$. To determine whether loss of $m$ Tep 1 could be essential for telomere length maintenance, we analyzed telomere length in mTep1-deficient mice and ES cells.

To examine telomere length in a total cell population, we used a quantitative method called Flow-FISH (43). We observed that none of the $m$ Tep $1^{+/-}$and three independent $m T e p 1^{-/-}$ES cell clones showed significant changes in average telomere fluorescence intensity, regardless of increasing population doublings, compared to early or late passages of wildtype cells (Fig. 3A). Thymocytes and splenocytes derived from different generations of $m$ Tep1-deficient mice (G1 to G7) also yielded very similar measurements of relative telomere lengths that were comparable to those of the G1 mTep1 heterozygote control (Fig. 3B and data not shown). The overall distribution of telomere signal fluorescence in the G1 and G7 samples was similar to that in the heterozygous mTepl control (data not shown). Similarly, FISH analysis of metaphase chromosome preparations from mTep1-deficient MEFs showed no significant change in telomere signal intensity or chromosomal aberrations compared to the wild-type MEFs (Fig. 4). We therefore conclude that disruption of $m$ Tepl had no effect on either the distribution or mean length of telomeres.

\section{DISCUSSION}

In mammals, the first genetic evidence for the essential role of telomerase activity in telomere length maintenance was elegantly demonstrated in mice lacking the telomerase RNA component $(2,14-16,29,39,42)$. In this study, we have used a genetic approach to examine the role of the first identified mammalian telomerase-associated protein, mTep1, in telomerase catalysis and telomere length regulation in vivo.

Despite the fact that TEP1 is associated with the telomerase RNA and the telomerase catalytic subunit TERT in cell extracts from immortalized human, mouse, and rat cells, mTep1deficient mice show no significant alteration in telomerase activity or telomere length. These results indicate that mTep1 is not essential for the catalytic activity of the telomerase complex, at least as measured by the in vitro elongation assay, and that as observed when deleted alone, mTep1 is not required for telomere length maintenance. These results are consistent with our previous finding from an in vitro assay that the reconsti- 


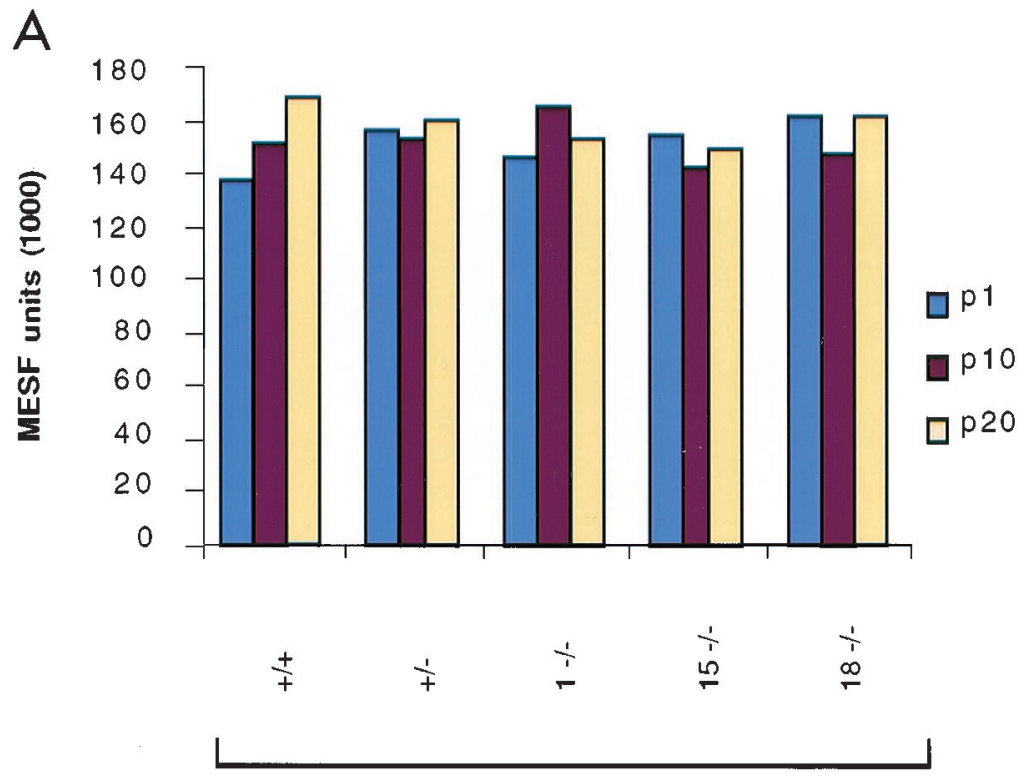

ES

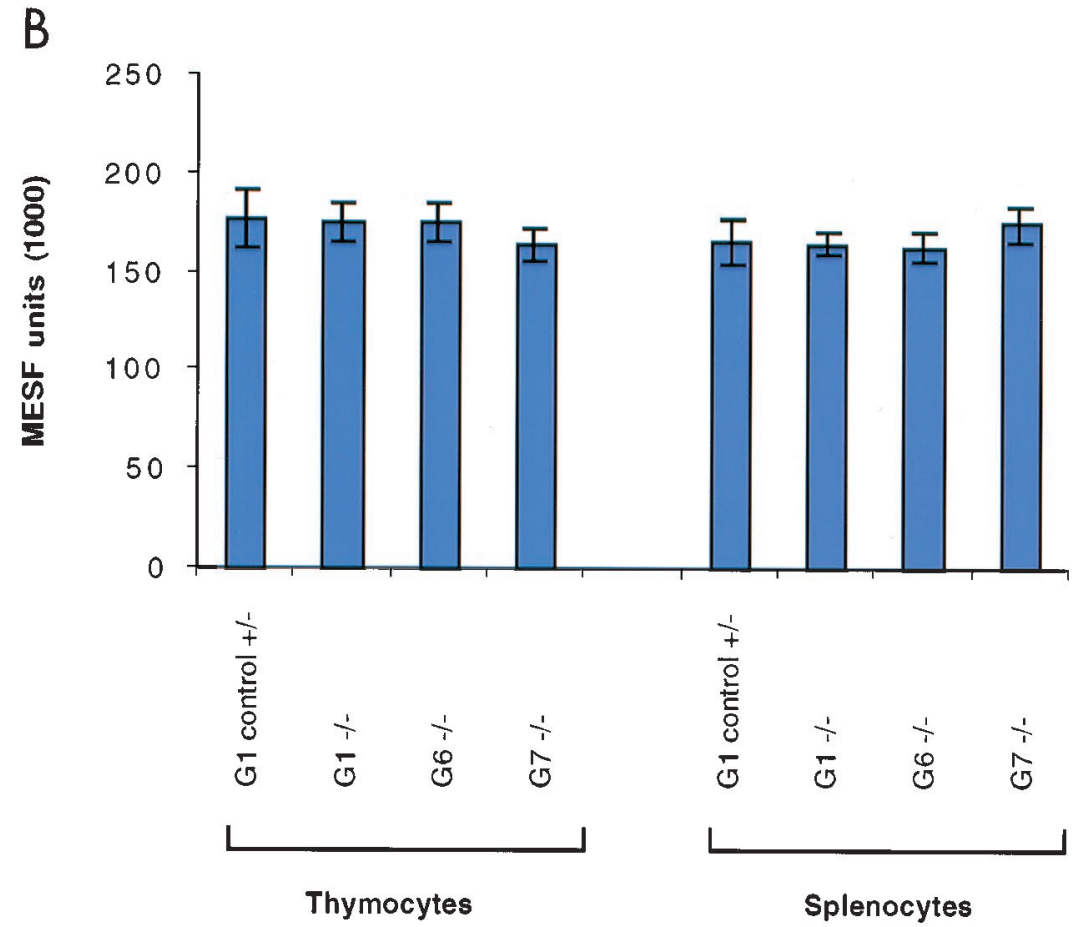

FIG. 3. Relative telomere lengths in ES cells and in splenocytes and thymocytes derived from mouse tissues, determined by Flow-FISH. (A) Average telomere fluorescence in early (p1) and later (p10 and p20) passages of ES cells, including wild type, $m$ Tep1 ${ }^{+/-}$, and three $m$ Tep1 ${ }^{-/-}$clones. Each MESF value represents the results for one individual ES clone at the indicated passage; hence, there are no error bars. (B) Average telomere fluorescence in splenocytes and thymocytes derived from early and late generations (G1 through G7) of $m$ Tep $1^{-1-}$ mice. In each set, data were pooled from at least five mice (error bars represent standard deviations).

tution of human telomerase activity does not require the addition of hTEP1 (1).

Several possibilities could account for the absence of a telomerase-associated phenotype in $m$ Tep $1^{-/-}$mice. Since mTep1 is associated with telomerase activity in immortalized cells, it is possible that it plays a specific role in the function of telome- rase in immortalized cells but not in normal cells (11). Another possibility is that if TEP1 is associated with only a fraction of the total telomerase activity in vivo, its disruption might have no overt phenotypic consequences. A substoichiometric association of TEP1 with telomerase activity is suggested by the observation that antibodies against TEP1, hStau, and L22 are 
wild type G1

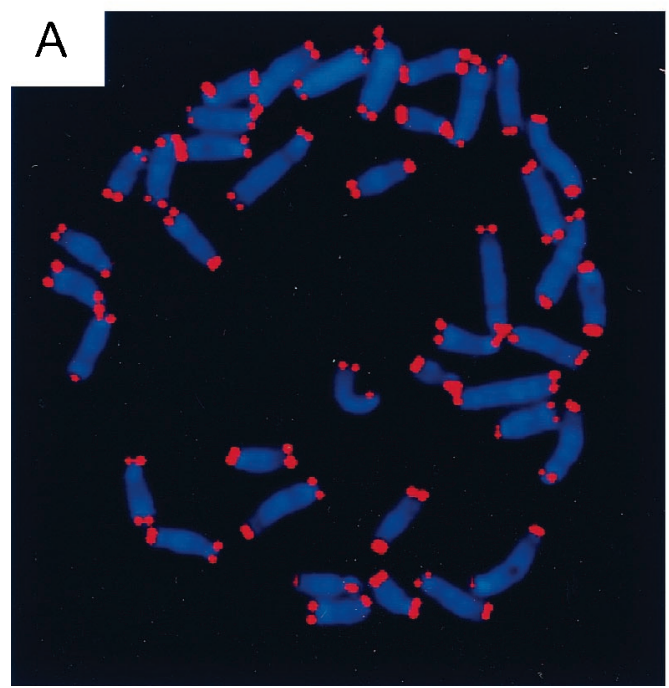

Tep1-/- G5

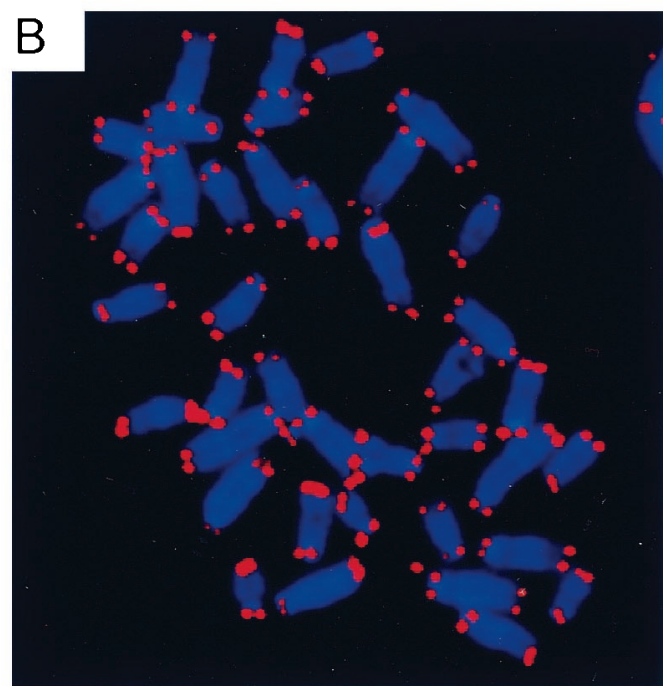

(A) or G5 $m \mathrm{mep}^{-1-}$ embryos (B). No significant changes in FIG. 4. FISH analysis of metaphase preparations of primary MEFs derived from G1 mTep $1^{+1}$
telomere signal intensity or chromosomal aberrations were detected in the G5 $\mathrm{mTep}^{-1-}$ embryos.

each able to immunoprecipitate telomerase activity, but not each other, from human immortalized cell extracts (28). Alternatively, other telomerase RNA binding proteins may share a redundant role with $\mathrm{mTep} 1$ in telomerase function. In Tetrahymena, both $\mathrm{p} 80$ and $\mathrm{p} 95$ synergistically bind to the telomerase RNA $(4,7)$. A mammalian homolog of p95 has not yet been identified. In mammals, two additional telomerase RNA binding proteins, L22 and hStau, were cloned using a yeast three-hybrid screen (28). These proteins are each associated with telomerase activity and hTERT, and yet there is no evidence of a direct association with each other or with TEP1 (28). Whether separate telomerase complexes containing distinct telomerase RNA binding proteins share overlapping functions in vivo has yet to be determined. Indirect immunofluorescence analysis of TEP1 and TERT will be instrumental in demonstrating whether TEP1 indeed colocalizes with a subset of hTERT in intact cells.

Not all telomerase-associated proteins appear to be restricted to the telomerase complex. At least some fraction of human telomerase activity is associated with p23 and Hsp90, two components of the ubiquitous foldosome which facilitate assembly of the active telomerase complex in rabbit reticulocyte lysates (18). TEP1 is expressed in many tissues, including those that lack telomerase activity, suggesting that TEP1 may be important for other cellular functions. In support of this notion, TEP1 has also recently been discovered to be an integral component of a large cytoplasmic (13-MDa) RNP complex referred to as the vault particle (24). Vault particles have been isolated from a number of organisms and appear to be ubiquitous among eukaryotes (19). Although the function of the vault particle is unknown, several recent reports have implicated the particle in intracellular transport $(3,17,20,27)$. Purified vaults display an eight-fold, barrel-like symmetry and contain at least three components in addition to TEP1, including the 104-kDa major vault protein MVP (21), a 193-kDa poly(ADP-ribosyl) polymerase called VPARP (23), and a small RNA called vRNA $(20,22)$. TEP1, but not telomerase activity, copurifies with the vault particle, and several vRNAs specifically interact with TEP1 in the yeast RNA-protein in- teraction assay (24). The so-called subunit sharing of the TEP1 protein between two seemingly unrelated RNPs suggests that TEP1 may play a more general role in RNP structure, function, or assembly. It is thus possible that TEP1 is a part of other RNPs in addition to the vault and telomerase complexes. Le and colleagues have also proposed that the telomerase RNA binding proteins hStau and L22 might be involved in aspects of RNA processing or RNP assembly that are not limited to telomerase (28). While the absence of a direct role for telomerase is confounding, the generation of $m$ Tepl-deficient mice is a first step toward the genetic dissection of the relationship between telomerase and the assembly and regulation of other cellular RNP complexes. We are currently examining mTep1deficient mice for the role of TEP1 in vaults and for the possible involvement of TEP1 in other RNPs. In addition, the generation of mice deficient in other telomerase-associated components will enable us to ascertain whether TEP1 is genetically redundant with other telomerase RNA binding proteins for telomerase function in vivo.

\section{ACKNOWLEDGMENTS}

We thank Carol Greider, Siyuan Le, Tak Mak, and members of the Harrington laboratory for critical comments and discussion; Denis Bouchard and Mark Ungrin for assistance with Flow-FISH; and Natalie Erdmann for technical assistance.

Y.L. is a research fellow of the National Cancer Institute of Canada and is supported with funds provided by the Terry Fox Run. L.H. acknowledges the support of the Medical Research Council and the National Institute of Health (no. AG8422117).

\section{REFERENCES}

1. Beattie, T. L., W. Zhou, M. O. Robinson, and L. Harrington. 1998. Reconstitution of human telomerase activity in vitro. Curr. Biol. 8:177-180.

2. Blasco, M. A., H. W. Lee, M. P. Hande, E. Samper, P. M. Lansdorp, R. A DePinho, and C. W. Greider. 1997. Telomere shortening and tumor formation by mouse cells lacking telomerase RNA. Cell 91:25-34.

3. Chugani, D. C., L. H. Rome, and N. L. Kedersha. 1993. Evidence that vault ribonucleoprotein particles localize to the nuclear pore complex. J. Cell Sci. 106:23-29. 
4. Collins, K., R. Kobayashi, and C. W. Greider. 1995. Purification of Tetrahymena telomerase and cloning of genes encoding the two protein components of the enzyme. Cell 81:677-686.

5. Counter, C. M., A. A. Avilion, C. E. LeFeuvre, N. G. Stewart, C. W. Greider, C. B. Harley, and S. Bacchetti. 1992. Telomere shortening associated with chromosome instability is arrested in immortal cells which express telomerase activity. EMBO J. 11:1921-1929.

6. Counter, C. M., M. Meyerson, E. N. Eaton, and R. A. Weinberg. 1997. The catalytic subunit of yeast telomerase. Proc. Natl. Acad. Sci. USA 94:92029207.

7. Gandhi, L., and K. Collins. 1998. Interaction of recombinant Tetrahymena telomerase proteins p 80 and p95 with telomerase RNA and telomeric DNA substrates. Genes Dev. 12:721-733.

8. Greider, C. W. 1996. Telomere length regulation. Annu. Rev. Biochem. 65:337-365.

9. Hakem, R., J. L. de la Pompa, C. Sirard, R. Mo, M. Woo, A. Hakem, A. Wakeham, J. Potter, A. Reitmair, F. Billia, E. Firpo, C. C. Hui, J. Roberts, J. Rossant, and T. W. Mak. 1996. The tumor suppressor gene Brca1 is required for embryonic cellular proliferation in the mouse. Cell 85:1009_ 1023

10. Harrington, L., C. Hull, J. Crittenden, and C. Greider. 1995. Gel shift and UV cross-linking analysis of Tetrahymena telomerase. J. Biol. Chem. 270: 8893-8901.

11. Harrington, L., T. McPhail, V. Mar, W. Zhou, R. Oulton, M. B. Bass, I. Arruda, and M. O. Robinson. 1997. A mammalian telomerase-associated protein. Science 275:973-977.

12. Harrington, L., W. Zhou, T. McPhail, R. Oulton, D. S. Yeung, V. Mar, M. B. Bass, and M. O. Robinson. 1997. Human telomerase contains evolutionarily conserved catalytic and structural subunits. Genes Dev. 11:3109-3115.

13. Henderson, L. O., G. E. Marti, A. Gaigalas, W. H. Hannon, and R. F. Vogt, Jr. 1998. Terminology and nomenclature for standardization in quantitative fluorescence cytometry. Cytometry 33:97-105.

14. Herrera, E., A. C. Martinez, and M. A. Blasco. 2000. Impaired germinal center reaction in mice with short telomeres. EMBO J. 19:472-481.

15. Herrera, E., E. Samper, and M. A. Blasco. 1999. Telomere shortening in $\mathrm{mTR}^{-1-}$ embryos is associated with failure to close the neural tube. EMBO J. 18:1172-1181.

16. Herrera, E., E. Samper, J. Martin-Caballero, J. M. Flores, H. W. Lee, and M. A. Blasco. 1999. Disease states associated with telomerase deficiency appear earlier in mice with short telomeres. EMBO J. 18:2950-2960.

17. Herrmann, C., E. Golkaramnay, E. Inman, L. Rome, and W. Volknandt. 1999. Recombinant major vault protein is targeted to neuritic tips of PC12 cells. J. Cell Biol. 144:1163-1172.

18. Holt, S. E., D. L. Aisner, J. Baur, V. M. Tesmer, M. Dy, M. Ouellette, J. B. Trager, G. B. Morin, D. O. Toft, J. W. Shay, W. E. Wright, and M. A. White. 1999. Functional requirement of p23 and Hsp90 in telomerase complexes. Genes Dev. 13:817-826.

19. Kedersha, N. L., M. C. Miquel, D. Bittner, and L. H. Rome. 1990. Vaults. II. Ribonucleoprotein structures are highly conserved among higher and lower eukaryotes. J. Cell Biol. 110:895-901.

20. Kickhoefer, V. A., K. S. Rajavel, G. L. Scheffer, W. S. Dalton, R. J. Scheper, and L. H. Rome. 1998. Vaults are up-regulated in multidrug-resistant cancer cell lines. J. Biol. Chem. 273:8971-8974.

21. Kickhoefer, V. A., and L. H. Rome. 1994. The sequence of a cDNA encoding the major vault protein from Rattus norvegicus. Gene 151:257-260.

22. Kickhoefer, V. A., R. P. Searles, N. L. Kedersha, M. E. Garber, D. L. Johnson, and L. H. Rome. 1993. Vault ribonucleoprotein particles from rat and bullfrog contain a related small RNA that is transcribed by RNA polymerase III. J. Biol. Chem. 268:7868-7873.

23. Kickhoefer, V. A., A. C. Siva, N. L. Kedersha, E. M. Inman, C. Ruland, M. Streuli, and L. H. Rome. 1999. The 193-kD vault protein, VPARP, is a novel poly(ADP-ribose) polymerase. J. Cell Biol. 146:917-928.

24. Kickhoefer, V. A., A. G. Stephen, L. Harrington, M. O. Robinson, and L. H. Rome. 1999. Vaults and telomerase share a common subunit, TEP1. J. Biol. Chem. 274:32712-32717.

25. Kilian, A., D. D. Bowtell, H. E. Abud, G. R. Hime, D. J. Venter, P. K. Keese, E. L. Duncan, R. R. Reddel, and R. A. Jefferson. 1997. Isolation of a candidate human telomerase catalytic subunit gene, which reveals complex splicing patterns in different cell types. Hum. Mol. Genet. 6:2011-2019.

26. Kim, N. W., M. A. Piatyszek, K. R. Prowse, C. B. Harley, M. D. West, P. L.
Ho, G. M. Coviello, W. E. Wright, S. L. Weinrich, and J. W. Shay. 1994. Specific association of human telomerase activity with immortal cells and cancer. Science 266:2011-2015.

27. Kong, L. B., A. C. Siva, L. H. Rome, and P. L. Stewart. 1999. Structure of the vault, a ubiquitous cellular component. Structure 7:371-379.

28. Le, S., R. Sternglanz, and C. W. Greider. 2000. Identification of two RNAbinding proteins associated with human telomerase RNA. Mol. Biol. Cell 11:999-1010.

29. Lee, H. W., M. A. Blasco, G. J. Gottlieb, J. W. Horner, II, C. W. Greider, and R. A. DePinho. 1998. Essential role of mouse telomerase in highly proliferative organs. Nature 392:569-574.

30. Lendvay, T. S., D. K. Morris, J. Sah, B. Balasubramanian, and V. Lundblad. 1996. Senescence mutants of Saccharomyces cerevisiae with a defect in telomere replication identify three additional EST genes. Genetics 144: 1399-1412.

31. Lingner, J., T. R. Cech, T. R. Hughes, and V. Lundblad. 1997. Three Ever Shorter Telomere (EST) genes are dispensable for in vitro yeast telomerase activity. Proc. Natl. Acad. Sci. USA 94:11190-11195.

32. Lingner, J., T. R. Hughes, A. Shevchenko, M. Mann, V. Lundblad, and T. R. Cech. 1997. Reverse transcriptase motifs in the catalytic subunit of telomerase. Science 276:561-567.

33. Lundblad, V., and J. W. Szostak. 1989. A mutant with a defect in telomere elongation leads to senescence in yeast. Cell 57:633-643.

34. Meyerson, M., C. M. Counter, E. N. Eaton, L. W. Ellisen, P. Steiner, S. D. Caddle, L. Ziaugra, R. L. Beijersbergen, M. J. Davidoff, Q. Liu, S. Bacchetti, D. A. Haber, and R. A. Weinberg. 1997. hEST2, the putative human telomerase catalytic subunit gene, is up-regulated in tumor cells and during immortalization. Cell 90:785-795.

35. Mitchell, J. R., E. Wood, and K. Collins. 1999. A telomerase component is defective in the human disease dyskeratosis congenita. Nature 402:551-555.

36. Nakamura, T. M., G. B. Morin, K. B. Chapman, S. L. Weinrich, W. H. Andrews, J. Lingner, C. B. Harley, and T. R. Cech. 1997. Telomerase catalytic subunit homologs from fission yeast and human. Science 277:955-959.

37. Nakayama, J., M. Saito, H. Nakamura, A. Matsuura, and F. Ishikawa. 1997. TLP1: a gene encoding a protein component of mammalian telomerase is a novel member of WD repeats family. Cell 88:875-884.

38. Nakayama, J., H. Tahara, E. Tahara, M. Saito, K. Ito, H. Nakamura, T. Nakanishi, T. Ide, and F. Ishikawa. 1998. Telomerase activation by hTRT in human normal fibroblasts and hepatocellular carcinomas. Nat. Genet. 18: 65-68.

39. Niida, H., T. Matsumoto, H. Satoh, M. Shiwa, Y. Tokutake, Y. Furuichi, and Y. Shinkai. 1998. Severe growth defect in mouse cells lacking the telomerase RNA component. Nat. Genet. 19:203-206.

40. Prowse, K. R., A. A. Avilion, and C. W. Greider. 1993. Identification of a nonprocessive telomerase activity from mouse cells. Proc. Natl. Acad. Sci. USA 90:1493-1497.

41. Prowse, K. R., and C. W. Greider. 1995. Developmental and tissue-specific regulation of mouse telomerase and telomere length. Proc. Natl. Acad. Sci. USA 92:4818-4822.

42. Rudolph, K. L., S. Chang, H. W. Lee, M. Blasco, G. J. Gottlieb, C. Greider, and R. A. DePinho. 1999. Longevity, stress response, and cancer in aging telomerase-deficient mice. Cell 96:701-712.

43. Rufer, N., W. Dragowska, G. Thornbury, E. Roosnek, and P. M. Lansdorp. 1998. Telomere length dynamics in human lymphocyte subpopulations measured by flow cytometry. Nat. Biotechnol. 16:743-747.

44. Seto, A. G., A. J. Zaug, S. G. Sobel, S. L. Wolin, and T. R. Cech. 1999. Saccharomyces cerevisiae telomerase is an Sm small nuclear ribonucleoprotein particle. Nature 401:177-180.

45. Singer, M. S., and D. E. Gottschling. 1994. TLC1: template RNA component of Saccharomyces cerevisiae telomerase. Science 266:404-409.

46. Weinrich, S. L., R. Pruzan, L. Ma, M. Ouellette, V. M. Tesmer, S. E. Holt, A. G. Bodnar, S. Lichtsteiner, N. W. Kim, J. B. Trager, R. D. Taylor, R. Carlos, W. H. Andrews, W. E. Wright, J. W. Shay, C. B. Harley, and G. B. Morin. 1997. Reconstitution of human telomerase with the template RNA component hTR and the catalytic protein subunit hTRT. Nat. Genet. 17: 498-502.

47. Zijlmans, J. M., U. M. Martens, S. S. Poon, A. K. Raap, H. J. Tanke, R. K. Ward, and P. M. Lansdorp. 1997. Telomeres in the mouse have large interchromosomal variations in the number of T2AG3 repeats. Proc. Natl. Acad. Sci. USA 94:7423-7428. 